\title{
Identification of two polypeptide segments of CCAAT/enhancer-binding protein required for transcriptional activation of the serum albumin gene
}

\author{
Alan D. Friedman and Steven L. McKnight \\ Howard Hughes Research Laboratories, Department of Embryology, Carnegie Institution of Washington, Baltimore, Maryland \\ 21210 USA
}

\begin{abstract}
We used molecular genetic methods to generate systematically altered forms of CCAAT/enhancer-binding protein (C/EBP). The aim of our experiments was to identify regions of C/EBP that contribute to its capacity to activate transcription from the promoter of the serum albumin gene in cultured hepatoma cells. Earlier experiments had shown that the DNA-binding domain must remain intact for C/EBP to activate albumin transcription. We now provide evidence of two additional elements of C/EBP that are required for its geneactivating role. One such element occurs within a 28-residue region located close to the amino terminus of the protein. The other maps to a broader, more internal region of the protein and appears to exhibit functional redundancy. These newly defined elements of C/EBP exhibit two characteristics of "activation" domains delineated in studies of other gene regulatory proteins. First, they play no obvious role in the capacity of C/EBP to bind to its DNA substrate. Second, they retain function after being appended onto the DNA-binding domain of a different protein. Neither of these putative activating elements is characterized by overt distinction in either charge or preponderance of any particular amino acid. The more amino-terminal element does, however, exhibit several features suggesting that it may assume an $\alpha$-helical structure. These studies offer observations and reagents that will be valuable for future studies concerning the physiologic function of C/EBP.
\end{abstract}

[Key Words: C/EBP; transcription; activation; serum albumin gene]

Received April 16, 1990; revised version accepted May 22, 1990.

During the past several years we have studied a heatstable DNA-binding protein initially prepared from rat liver nuclei (Graves et al. 1986; Johnson et al. 1987). This protein, termed CCAAT/enhancer-binding protein (C/EBP), binds tightly to DNA sequences that commonly occur in the promoters and enhancers of mRNAcoding genes. It was therefore anticipated to represent a transcription factor. The gene for C/EBP has been isolated, and various antisera specific to the protein have been raised (Landschulz et al. 1988a). These reagents have allowed the completion of a variety of descriptive experiments on the nature of C/EBP.

Expression of $\mathrm{C} / \mathrm{EBP}$ is tissue restricted (Birkenmeier et al. 1989). In the adult mouse, C/EBP mRNA is most abundant in liver and adipose tissue. C/EBP expression is limited further to the terminally differentiated cells most responsible for performing the specialized physiological roles of these tissues. In liver, for example, it is restricted to parenchymal cells. Likewise, cultured 3T3-L1 preadipocytes only express C/EBP when induced to assume the fully differentiated state. Finally, Friedman et al. (1989) reported that neoplastically transformed liver cells contain substantially reduced levels of
C/EBP relative to the parenchymal cells from which they derive. These observations led to the hypothesis that C/EBP might play a general role in establishing and maintaining the differentiated, nonproliferative state.

Consistent with the idea that C/EBP regulates transcription as a function of terminal cell differentiation are observations that it activates the promoters of the serum albumin gene (Friedman et al. 1989), the steroylCoA desaturase gene (Christy et al. 1989), the insulin-responsive glucose transporter gene (Kaestner et al. 1990), and the phosphoenolpyruvate carboxykinase (PEPCK) gene (Trus et al. 1990; Park et al. 1990). All of these genes encode products that are expressed primarily in differentiated cells. C/EBP's unusual tissue distribution, repertoire of target genes, and concentration fluctuation in adult liver (Birkenmeier et al. 1989) led to the hypothesis that it might play a central role in regulating energy metabolism (McKnight et al. 1989). This hypothesis anticipates that the level and/or activity of $\mathrm{C} / \mathrm{EBP}$ will be regulated, perhaps hormonally, as a function of the energy status of the animal.

The observations and ideas outlined in the preceding paragraphs indicate that C/EBP may have several func- 
tions-including gene-activating roles in several specialized tissues, a general role in cellular growth inhibition, and a hypothetical responsiveness to hormones that modulate energy balance. A principal goal of our studies has been to connect these presumed physiological functions of C/EBP with defined aspects of its polypeptide structure.

Some progress has been made on discovering how the nature of the domain of C/EBP allows it to bind DNA. Upon determining the amino acid sequence of $C / E B P$, similarities were observed between its sequence and the sequences of two transforming proteins, Myc and Fos (Landschulz et al. 1988a). Knowing that these regions of sequence relatedness were localized to the DNA-binding domain of C/EBP, it was reasoned that they might reflect the underpinnings of a structural motif designed to facilitate specific interaction between protein and DNA. By studying the amino acid sequences shared among these proteins and conducting molecular genetic and biochemical experiments on C/EBP, a relatively simple model was developed that may account for a common three-dimensional shape that these proteins adopt upon binding DNA (Landschulz et al. 1988b, 1989; Vinson et al. 1989). According to this model, the DNA-binding domain of C/EBP is composed of two contiguous components, a dimerization interface termed the leucine zipper, and a DNA contact surface termed the basic region.

The DNA-binding domain of C/EBP, which is localized within the carboxy-terminal 80 residues of the 359amino-acid polypeptide, must remain intact for the protein to activate transcription in a gene-specific manner (Friedman et al. 1989). In this study, we conducted experiments that investigate whether additional aminoterminal segments might contribute to the gene-activating role of C/EBP. Our results provide evidence of several polypeptide segments that perform an important role in the process of gene activation, yet, at least as tested in vitro, are not required for DNA binding. These results, in distinguishing polypeptide determinants necessary for gene activation from those required for DNA binding, are consistent with numerous earlier studies (Hochschild et al. 1983; Hope and Struhl 1986; Ma and Ptashne 1987a). By delineating additional, functionally important regions of the polypeptide, the present studies may help bridge the gap in understanding between the physiology and molecular biology of C/EBP.

\section{Results}

\section{$A$ discrete activation element is located close to the amino terminus of $C / E B P$}

To search for regions of the C/EBP polypeptide that might play a role in transcriptional activation, we employed molecular genetic methods to delete segments of the encoding gene. Deletions were introduced into an expression vector, pMSV-C/EBP-wt, that is capable of directing synthesis of intact C/EBP in cultured mammalian cells (Friedman et al. 1989). Our strategy was to remove sequential blocks of amino acids, each $\sim 30$ res- idues in length. We chose prolines as boundary residues for each deletion and replaced each deleted block with the dipeptide sequence glycine-threonine, as encoded by the hexanucleotide sequence GGTACC. This sequence specifies a KpnI restriction endonuclease recognition site, which later facilitated the creation of larger deletions. The experimental procedures that were used to generate deleted variants of the C/EBP gene are outlined in Materials and methods.

Figure 1A shows a diagrammatic representation of the nine initial deletions that were created. Deleted variants were numbered $\Delta 1-\Delta 9$, with the $\Delta 1$ deletion being closest to the amino terminus of the protein and $\Delta 9$ being closest to the DNA-binding domain. A recombinant between the genes encoding $\Delta 1$ and $\Delta 9$, termed $\Delta 1-9$ removed a 247 -amino-acid region covering most of the C/EBP protein other than its DNA-binding domain. A list of the exact end points of each deletion mutant is presented in the legend to Figure 1.

Our initial characterization of these foreshortened C/EBPs measured their expression level relative to that of intact C/EBP in cultured HepG2 cells. Individual cultures of HepG2 cells were transfected by the calcium phosphate method, with an expression vector encoding each deletion mutant. Two days later, total cellular protein was isolated and analyzed by Western blotting, with an antiserum specific to the carboxy-terminal 15 residues of C/EBP. According to this assay, the deleted variants of $\mathrm{C} / \mathrm{EBP}$ fell into three categories. Two variants, $\Delta 1$ and $\Delta 2$, produced no cross-reacting material. Two others, $\Delta 9$ and $\Delta 1-9$, produced approximately onetenth the level of cross-reacting material compared with intact C/EBP. The six remaining variants produced amounts of cross-reacting material that were approximately equivalent to the normal protein. This series of experiments was repeated on three occasions, and in each trial, very similar patterns of expression were observed. Finally, the DNA sequences of the genes encoding the $\Delta 1$ and $\Delta 2$ variants were resolved to ensure that their inability to be expressed was not related to unanticipated disruptions in the C/EBP open reading frame that might have occurred during mutagenesis.

We then examined the subcellular localization of each variant protein. Cultures of HepG2 cells were transfected with expression vectors specifying the synthesis of each of the aforementioned variant polypeptides. After a 24-hr incubation interval, the cells were trypsinized and replated into culture dishes containing glass coverslips. The intracellular location of C/EBP was examined by indirect immunofluorescence 2 days after transfection (Fig. 1C). No positive cells were observed in the case of the $\Delta 2$ variant. Very weak staining was observed in the nuclei of $\sim 0.1 \%$ of the cells that had been transfected with the $\Delta 1$ variant. All remaining variants, including $\Delta 1-9$, showed an intense, nuclear staining pattern in $\sim 2 \%$ of the cells. This latter pattern was indistinguishable from that generated by intact C/EBP.

As a final assay, each expression vector was cotransfected into HepG2 cells along with a chimeric target gene designated pAT2. This target gene consists of the 
$1 \mathrm{~A}$

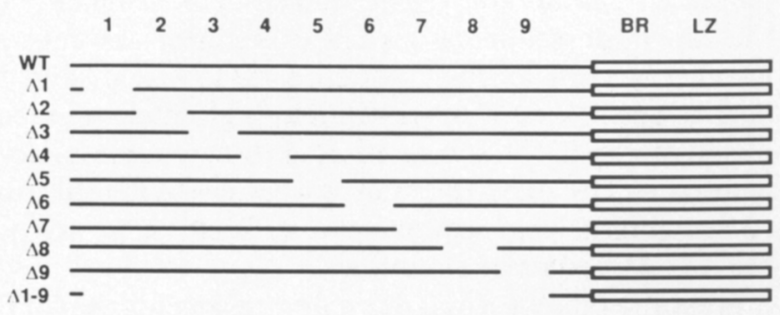

$1 \mathrm{C}$
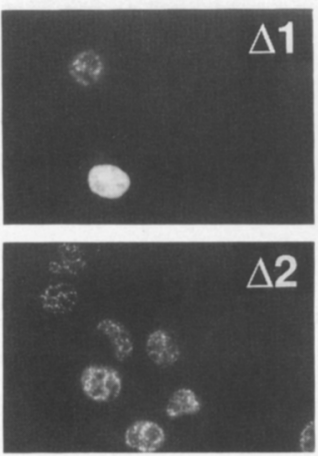

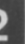
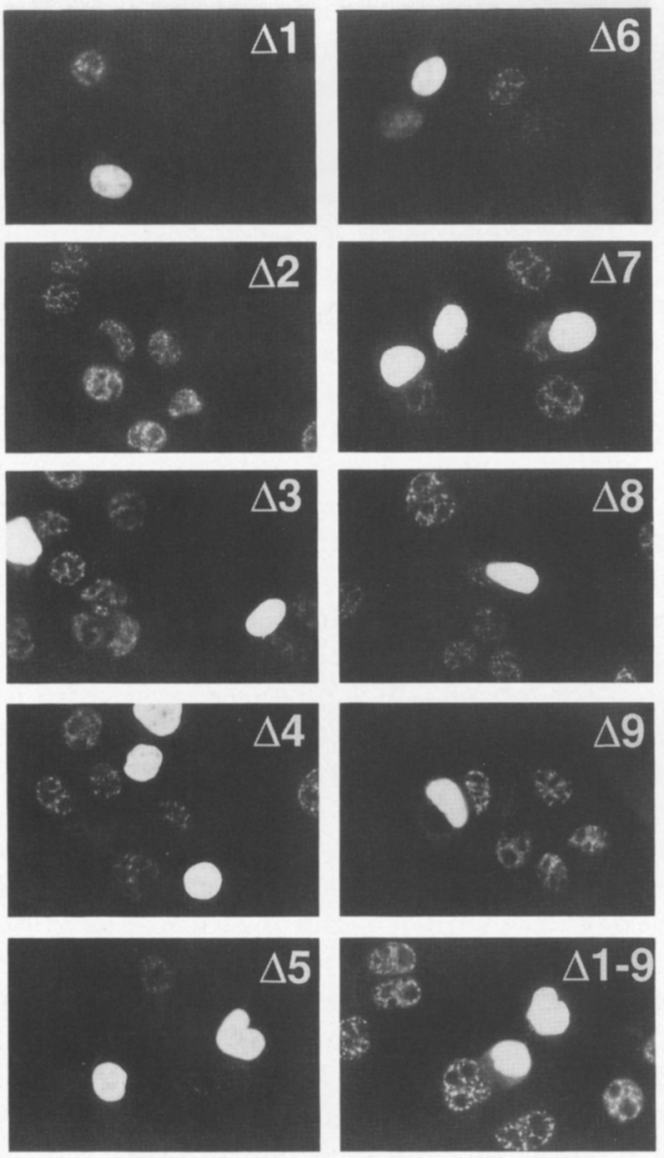

1B

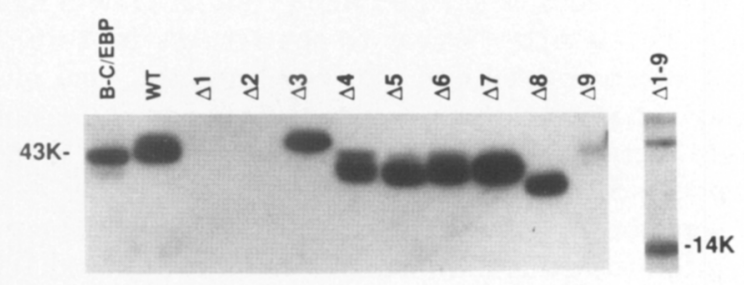

1D

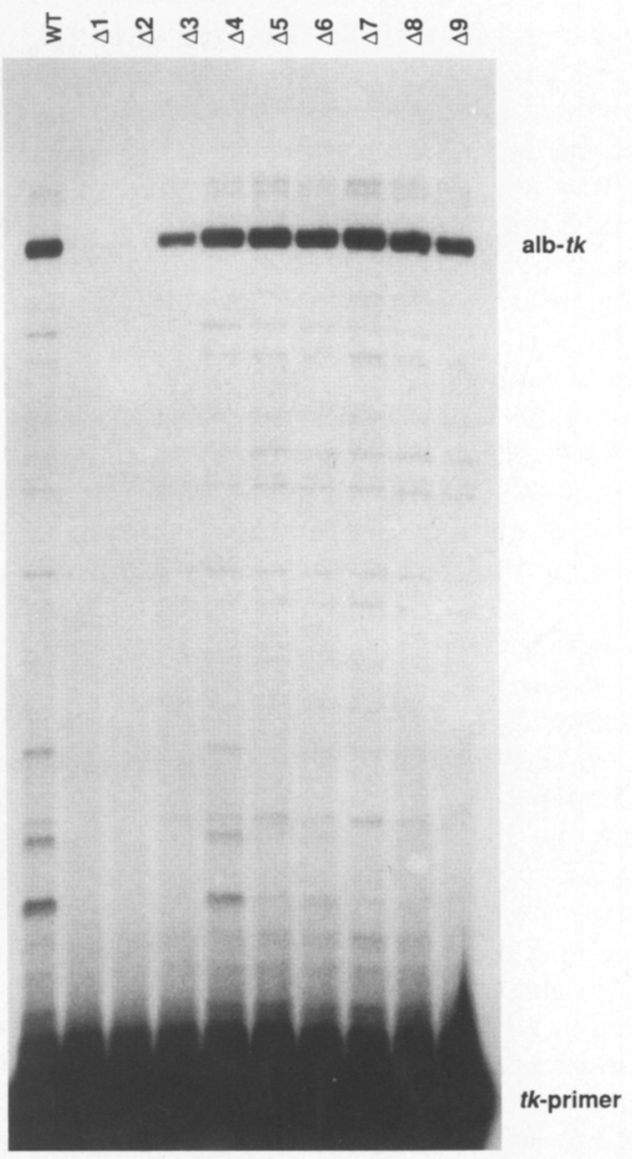

Figure 1. (See facing page for legend.)

promoter of the mouse serum albumin gene linked to the mRNA-coding element of the herpes simplex virus thymidine kinase $(t k)$ gene (K. Zaret, unpubl.). Earlier experiments had shown that C/EBP binds avidly to the promoter of the albumin gene (Cereghini et al. 1987; Costa et al. 1988; Maire et al. 1989) and can activate its transcription in transiently transfected HepG2 cells (Friedman et al. 1989). Total cellular RNA was isolated from each culture 2 days after transfection, and expression of $t k$ mRNA was monitored by primer extension.

Previous experiments had shown that cotransfection of pAT2 with the expression vector that specifies synthesis of native C/EBP (pMSV-C/EBP-wt) results in po- tent trans-activation (Friedman et al. 1989). Quantitation of the fold induction generated by C/EBP is difficult to assess because little or no $t k$ mRNA can be detected when cells are transfected with pAT2 alone (Friedman et al. 1989). However, in the case of the present studies, we were able to compare the expression levels induced by various mutated forms of C/EBP (Fig. 1D). As expected from the results of Western blotting (Fig. 1B), neither $\Delta 1$ nor $\Delta 2$ was able to induce expression from pAT2. On the other hand, the $\Delta 9$ variant, which was expressed at only one-tenth the normal level according to Western blotting, showed surprising potency in the trans-activation assay. One of the remaining variants, $\Delta 3$, reproducibly 
exhibited a decrease of approximately fourfold in transactivating capacity. The $\Delta 4-\Delta 8$ variants exhibited activities indistinguishable from native C/EBP. Finally, no trans-activation capacity was observed in the case of the deletion mutant that removed all nine blocks of amino acids $(\Delta 1-9)$.

We make note of the observation that the $\Delta 9$ variant was almost fully competent in the trans-activating assay (Fig. 1D), despite being expressed at only one-tenth the normal level (Fig. 1C). This observation indicates that the subpopulation of cells that take up DNA in the transient transfection assay express saturating levels of C/EBP (except in the cases of $\Delta 1$ and $\Delta 2$ ). It would thus appear that the $\Delta 1-9$ variant, which is expressed at an equivalent level to $\Delta 9$ (Fig. 1C), is genuinely impaired in its capacity to activate transcription from the albumin promoter.

In an attempt to assess further the roles of regions $1-3$, two larger deletion mutants were prepared. By using the unique $K p n I$ restriction site present in the encoding gene of each variant, $\Delta 1$ was recombined with both $\Delta 2$ and $\Delta 3$, leading to $\Delta 1-2$ and $\Delta 1-3$ (Fig. 2A). We reproducibly observed that both of these new variants were expressed at a higher than normal level in transfected HepG2 cells (Fig. 2B). On all occasions tested, the protein product of $\Delta 1-3$ was observed to exceed that produced from the intact C/EBP gene by at least a fivefold level. When compared with intact C/EBP in the transient trans-activation assay, $\Delta 1-2$ was slightly more active than normal, whereas $\Delta 1-3$ was substantially impaired. These data provide evidence that regions 1 and 2 may not play a critical role in the capacity of C/EBP to activate transcription from the albumin promoter. Furthermore, they provide additional evidence that region 3 may play an important role in the activation process.

\section{The central region of C/EBP contains a redundant set of activating elements}

Although both the $\Delta 3$ and $\Delta 1-3$ variants of C/EBP were markedly weakened in their capacity to activate the albumin promoter, they did retain a measurable activity level relative, for example, to $\Delta 1-9$. The discrepant activities of these deleted proteins raised the possibility that the amino acid sequences encoded by regions 4-9 might play a role in transcriptional activation that could not be discerned by the simple removal of 30-amino-acid blocks (Fig. 1A). To examine this possibility, we prepared a series of larger deletions (Fig. 3A). The first six of these larger deletions, $\Delta 3-4, \Delta 3-5, \Delta 3-6, \Delta 3-7, \Delta 3-8$, and $\Delta 3-9$, as well as $\Delta 3$, all lacked region 3 . The other five larger deletions, $\Delta 4-9, \Delta 5-9, \Delta 6-9, \Delta 7-9$, and $\Delta 8-9$, as well as $\Delta 9$, all retained region 3 and added to it successively larger portions of regions 4-9.

Before testing the activities of these new variant forms of $C / E B P$, their expression levels were examined by Western blotting. As shown in Figure 3B, all 13 variants were expressed at levels similar to, or greater than, native C/EBP. Although expressed at a consistently high level, certain variants exhibited anomalous electrophoretic mobilities. This was most evident in the series of proteins that retained a common deletion end point corresponding to the amino terminus of region 3 and can be noticed by saltatory jumps in electrophoretic mobility as the proteins are compared side by side in Western blots (Fig. 3B). Indeed, two of the proteins, $\Delta 3-5$, and $\Delta 3-7$, resolved into two closely migrating bands when sized on SDS-polyacrylamide gels. In contrast, the series of variants that were designed to retain a common deletion end point at the carboxyl terminus of region 9 formed the relatively smooth progression of electrophoretic mobility changes expected from the amount of encoding sequence that had been removed.

Expression vectors specifying the synthesis of each protein schematized in Figure $3 \mathrm{~A}$ were cotransfected with pAT2 into HepG2 cells. The results of these transactivation assays are shown in Figure 3C. Relative to the $\Delta 3$ variant, gradedly reduced levels of activity were observed for $\Delta 3-5$ and $\Delta 3-6$. No activity was observed for $\Delta 3-7, \Delta 3-8$, and $\Delta 3-9$. Interpreted most literally, these

Figure 1. Expression level and activity measurements of deleted variants of C/EBP. (A) Schematic diagram of native C/EBP (top) and deleted forms of the protein. The nomenclature of each deletion mutant is shown at left. The protein segment deleted is indicated by a discontinuity in the horizontal line corresponding to an amino-to-carboxyl representation of the $43-\mathrm{kD} \mathrm{C} / \mathrm{EBP}$ polypeptide. The DNA-binding domain is shown as a rectangular box consisting of basic region (BR) and leucine zipper (LZ). Arabic numerals 1-9 along the top indicate the contiguous regions that were individually deleted. The two end points of each deletion corresponded to the positions of proline residues native to the C/EBP amino acid sequence. The nine original deletions were prepared by using end points dictated by proline residues located at amino acids $11,43,70,97,127,155,178,200,235$, and 257 (see Landschulz et al. 1988a). (B) Western blot assays used to monitor the expression of native and variant forms of C/EBP. Monolayer cultures of HepG2 cells were transiently transfected with $10 \mu \mathrm{g}$ of each expression vector, along with $15 \mu \mathrm{g}$ of sonicated salmon sperm DNA. Total cell protein was harvested $40 \mathrm{hr}$ later. Protein samples were sized on an SDS-polyacrylamide gel, transferred to nitrocellulose, and probed with an antiserum specific to the carboxyl terminus of C/EBP (Materials and methods). The left-most lane was loaded with C/EBP that had been expressed in bacterial cells. Remaining lanes were loaded with protein from HepG2 cells that had been transfected with either native C/EBP or one of the deleted forms shown in $A .|C|$ Immunofluorescence staining patterns of HepG2 cells that had been transfected with native and variant forms of C/EBP. Cells were fixed for $40 \mathrm{hr}$ after initial exposure to DNA and processed for indirect immunofluorescence as described in Materials and methods. $(D)$ Transient activation assays of the albumin promoter by native and variant forms of C/EBP. Cultured HepG2 cells were transfected with $10 \mu \mathrm{g}$ of pAT2, which contains the promoter of the mouse serum albumin gene linked to the herpes simplex virus $t k$ gene, $5 \mu \mathrm{g}$ of sonicated salmon sperm DNA, and $10 \mu \mathrm{g}$ of a plasmid encoding either native C/EBP or one of the nine mutated variants shown in $A$. Forty hours after transfection, cells were harvested and RNA was purified. Expression of pAT2 was determined by primer extension analysis with a synthetic oligonucleotide complementary to tk mRNA (see Materials and methods). The positions of the fully extended primer (alb-tk) and unreacted primer ( $t k$-primer) are indicated at right. 


\section{A}
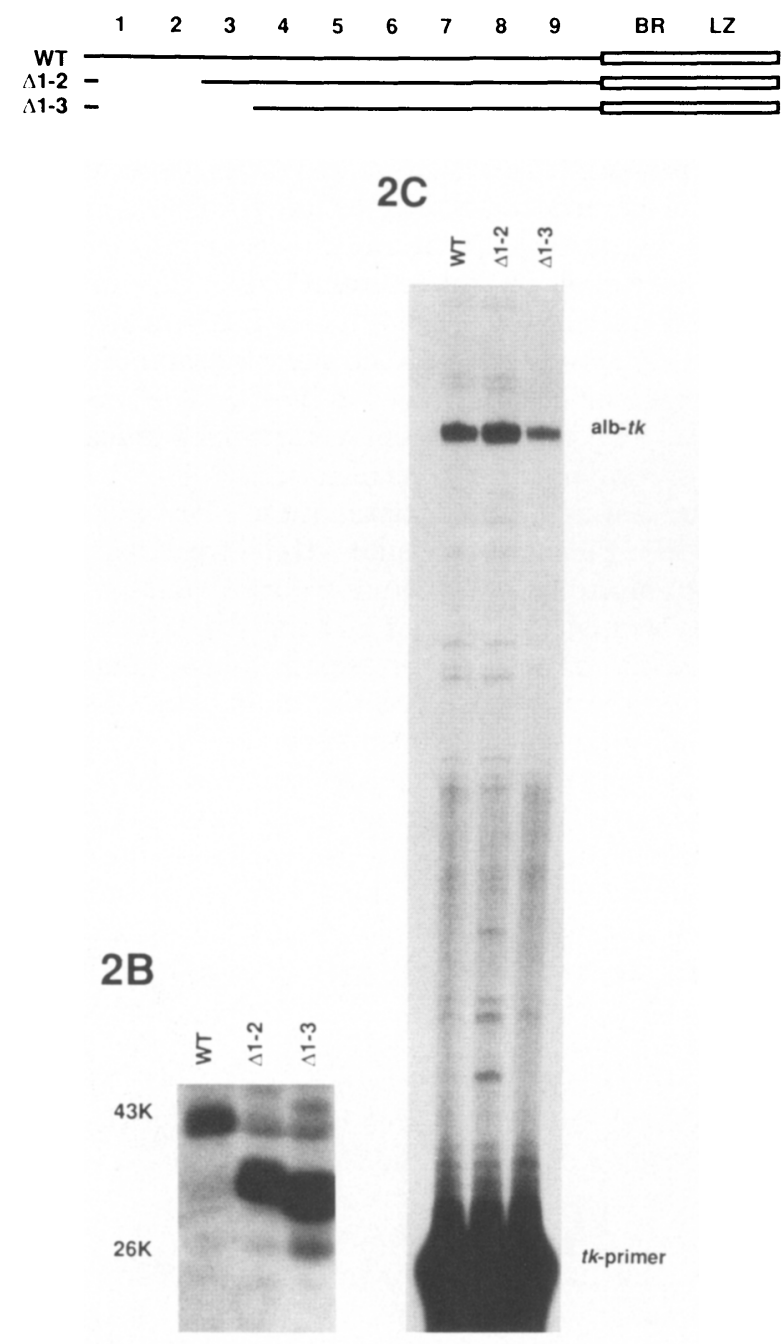

Figure 2. Expression level and activity measurements of deleted variants of C/EBP. $(A)$ Diagram of native C/EBP and variant forms that remove regions $1-2$ and $1-3 .(B)$ Western blot assays for expression of C/EBP forms shown in $A$. $(C)$ Transient activation assays of the albumin promoter by C/EBP forms shown in $A$.

data reveal a redundant "activation" component located in regions 5-7.

Other than the DNA-binding domain of C/EBP, the $\Delta 3-9$ variant retains only regions 1 and 2 . Despite being expressed at a high level, $\Delta 3-9$ is inactive. When region 3 was added back to the $\Delta 3-9$ variant, creating $\Delta 4-9$, significant trans-activation capacity was restoredagain emphasizing the importance of region 3 (Fig. 3C). The further addition of region 4 did not increase transactivation capacity, consistent with the observation that its removal in the progression from $\Delta 3$ to $\Delta 3-4$ had minimal effect (Fig. 3C). Addition of region 5, on the other hand, restored trans-activation capacity to the level of the native protein, again consistent with the observation that its removal in the progression from $\Delta 3-4$ to $\Delta 3-5$ diminished trans-activation. Further addition of regions 6-9 did not improve activation potential significantly.

$A$ derivative of $C / E B P$ containing the leucine zipper of GCN4 is functionally active

A chimeric protein in which the leucine zipper of C/EBP was replaced by the analogous region from GCN4 has been described (Agre et al. 1989). This protein, diagrammed schematically in Figure 4A, was shown to retain DNA-binding properties indistinguishable from native C/EBP. A DNA fragment encompassing this chimeric protein was transferred into the C/EBP expression vector, allowing synthesis of a protein termed C/EBP-GZ (see Materials and methods). This protein was expressed in transiently transfected HepG2 cells at a level similar to native C/EBP (Fig. 4B). The protein was also capable of activating transcription from the albumin promoter as tested on the pAT2 target gene (Fig. 4C). Apparently, under these assay conditions, the leucine zipper of GCN4 can functionally replace that of C/EBP.

The region 1-3 and 4-10 activating elements maintain function after transfer to a heterologous DNA-binding domain

Chimeric proteins were prepared by linking various segments of C/EBP onto the DNA-binding domain of GAL4 (Laughon and Gesteland 1984; Keegan et al. 1986; Ma and Ptashne 1987b; Sadowski and Ptashne 1989). The segments of C/EBP that were used include regions 1-9, $1-2,1-3,3$ alone, 3-9, and 4-9 (Fig. 5A; see also Materials and methods|. Only three of the chimeric proteins, GAL1-9, GAL3-9, and GAL4-9 (see Fig. 5A), contained epitopes that could be detected by available C/EBP-specific antisera. Expression was monitored in HepG2 cells by Western blotting (Fig. 5A). Cross-reactive proteins of the expected size were observed in all three cases; however, more rapidly migrating polypeptide species were also observed, perhaps indicative of partial proteolytic degradation.

Because GAL4 and C/EBP bind to different DNA sequences, a target gene capable of binding the GAL4-C/EBP chimeric proteins was prepared. The C/EBP-binding site located $100 \mathrm{bp}$ upstream from the albumin mRNA start site, which had been shown previously to be critical for C/EBP-mediated trans-activation (Friedman et al. 1989), was deleted and replaced with four avid GAL4-binding sites (see Materials and methods).

This modified form of pAT2, termed pAT2-GAL, was cotransfected into HepG2 cells along with one of the aforementioned expression vectors. Transfection of pAT2-GAL, as with pAT2, resulted in no detectable expression (data not shown). The vector that encodes the DNA-binding domain of GAL4 caused a slight increase in the synthesis of $t k$ mRNA. Conversely, the GAL1-9 expression vector led to a considerably higher level of transcription from the derivatized albumin promoter.

The remaining chimeric proteins contained various 
parts of the amino-terminal region of $C / E B P$ that were suspected to function in the process of transcriptional activation. Regions 1 and 2, when appended onto the GAL4 DNA-binding domain alone, failed to improve the level of trans-activation. However, when region 3 was included, potent activation was observed. These results demonstrated that regions $1-3$ of C/EBP retain transcriptional activation function after being transferred onto the DNA-binding domain of a heterologous protein. They also confirmed the critical nature of region 3. Finally, these observations demonstrated that a small, amino-terminal segment of C/EBP can potently activate a derivatized form of the albumin promoter in the complete absence of the C/EBP DNA-binding domain.

Paradoxically, region 3 alone did not confer activating function when appended to the GAL4 DNA-binding domain (cf. GAL1-3 with GAL3). We were surprised that the removal of regions 1 and 2 would cause so dramatic an effect, as deletion of these regions from otherwise intact C/EBP did not reduce its activity (Fig. 2). It is pos- sible that the GAL3 chimeric protein is unstable. Alternatively, the activity of region 3 might be occluded when it is linked too closely to the GAL4 DNA-binding domain.

A comparison of the final two derivatives provided results pertinent to this question. The GAL3-9 and GAL4-9 chimeras led to equivalent, intermediate levels of trans-activation. These results demonstrated the capacity of regions 4-9 to activate transcription when appended to a heterologous DNA-binding domain. Moreover, they provided evidence that region 3 functions poorly when directly abutted to the GAL4 DNA-binding domain. That is, the addition of region 3 onto GAL4-9 failed to improve its activation function (Fig. 5C) despite being expressed at an equivalent level (Fig. 5B).

\section{Discussion}

The results outlined in this report indicate that C/EBP contains several distinguishable polypeptide elements

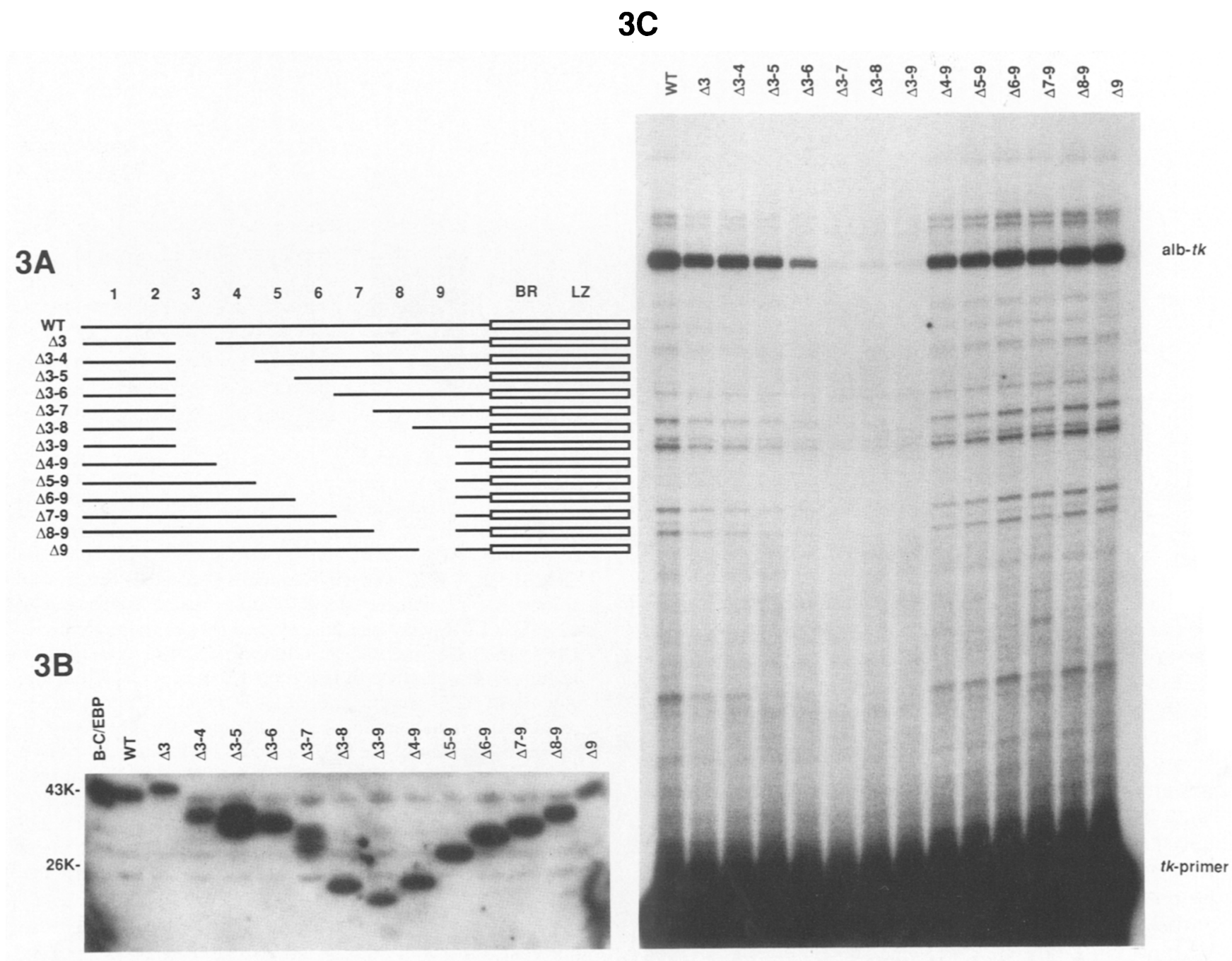

Figure 3. Expression level and activity measurements of deleted variants of C/EBP. $(A)$ Diagram of native C/EBP and variant forms that remove gradedly larger segments of the central region of the protein. $(B)$ Western blot assays for expression of C/EBP forms shown in $A$. $(C)$ Transient activation assays of the albumin promoter by C/EBP forms shown in $A$. 
$4 A$
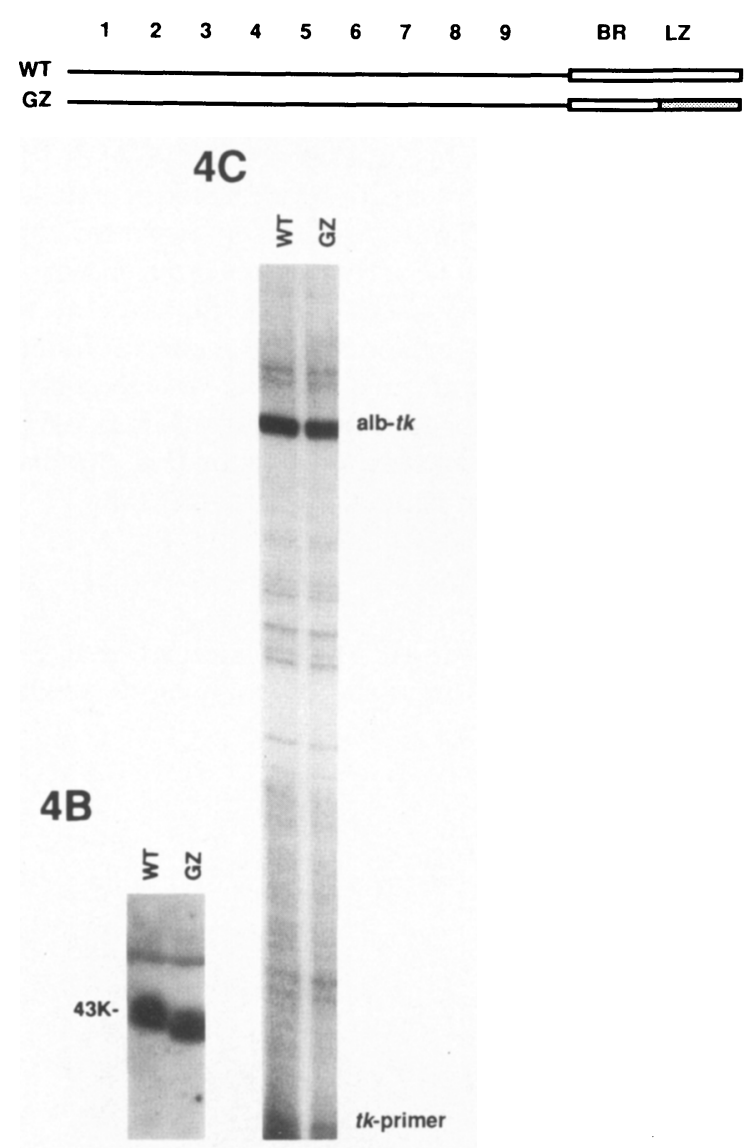

Figure 4. Expression level and activity measurements of a C/EBP-GCN4 hybrid protein. $(A)$ Diagram of native C/EBP and the hybrid protein C/EBP-GZ. The hybrid protein replaces the leucine zipper of C/EBP with that of CGN4 as described by Agre et al. (1989). (B) Western blot assays for the expression of C/EBP and C/EBP-GZ. The antiserum used to detect both proteins was directed against a 14-amino-acid epitope located on the immediate amino-terminal side of the C/EBP DNA-binding domain (Landschulz et al. 1988). (C) Transient activation assays of the albumin promoter by C/EBP and C/EBP-GZ.

that are required for efficient trans-activation of the serum albumin promoter yet are dispensable for avid interaction with DNA. Similar elements have been identified in a number of other regulatory proteins that bind DNA sequence specifically, including GCN4, GAL4, SP1, cJUN, CTF, OCT-2, and CREB (Hope and Struhl 1986; Ma and Ptashne 1987a; Courey and Tjian 1988; Bohmann and Tjian 1989; Mermod et al. 1989; Tanaka and Herr 1990; Yamamoto et al. 1990). Such elements have been termed "activating domains," consistent with the anticipation that they might directly stimulate the process of transcription initiation.

We provisionally identify two activating domains within C/EBP (Fig. 6). One, termed region 3, is located relatively close to the amino terminus of C/EBP. When region 3 was deleted from an otherwise intact form of C/EBP, trans-activation potential was reduced to a level

\section{A}

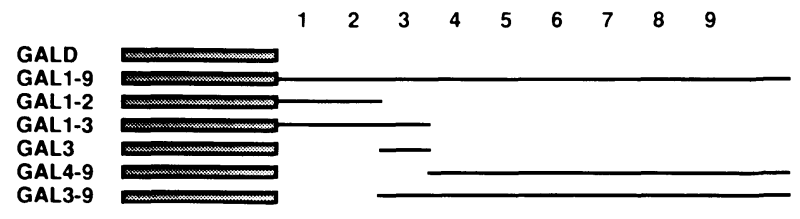

$5 \mathrm{C}$

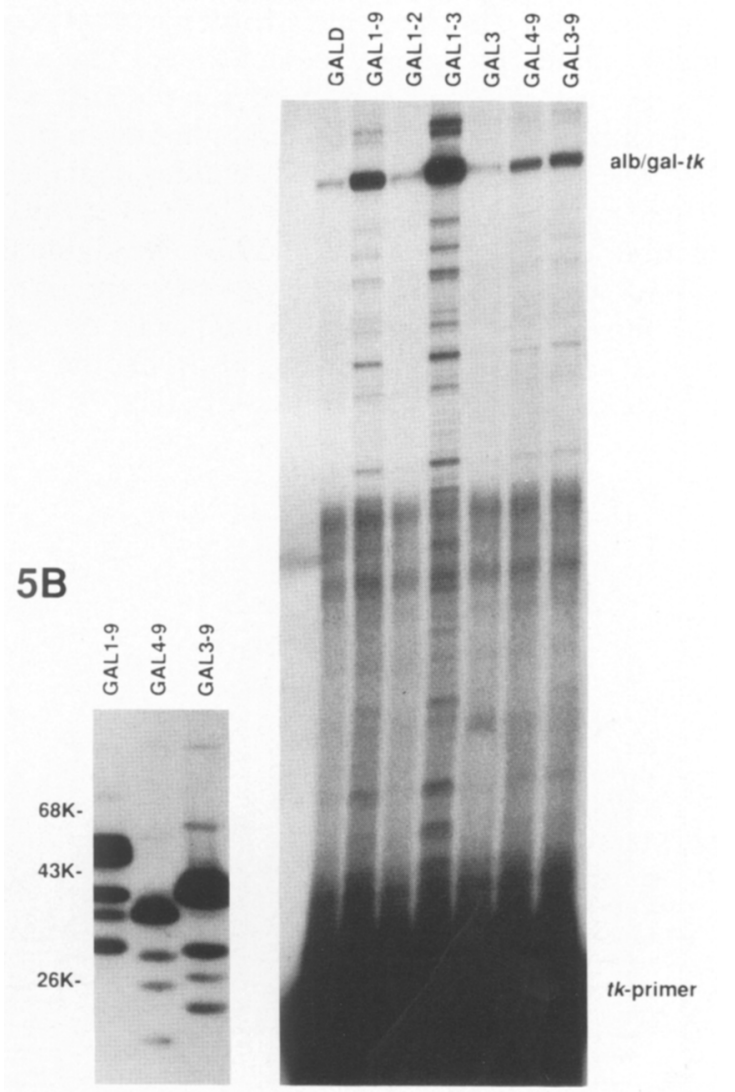

Figure 5. Expression level and activity measurements of GAL4-C/EBP hybrid proteins. (A) Diagram of native C/EBP and hybrid proteins that contain various segments of $C / E B P$ linked to the DNA-binding domain of GAL4. Stippled rectangles correspond to amino acids 1-147 of GAL4 protein (Laughon and Gesteland 1984). The amino-terminal 147 residues of GAL4 are sufficient for sequence-specific DNA binding (Keegan et al. 1986). GALD corresponds to a protein that contains the GAL4 DNA-binding domain alone. Numbers above $A$ correspond to regions 1-9, as defined in Fig. 1. GAL1-9 contains all nine regions of C/EBP linked to the GAL4 DNA-binding domain. Remaining proteins contain various regions of C/EBP linked to the same segment of the GAL4 protein. $(B)$ Western blot assays for the expression of GAL4-C/EBP fusion proteins. The antiserum used to detect fusion proteins was directed against a 14amino-acid epitope located within region 9 of C/EBP (Landschulz et al. 1988a). (C) Transient activation assays of the albumin promoter by GALD and GAL4-C/EBP fusion proteins.

between $20 \%$ and $30 \%$ of normal. On its own, region 3 was unable to confer activating potential to the DNAbinding domain of GAL4; however, when linked to GAL4 along with the preceding 69 amino acids of 


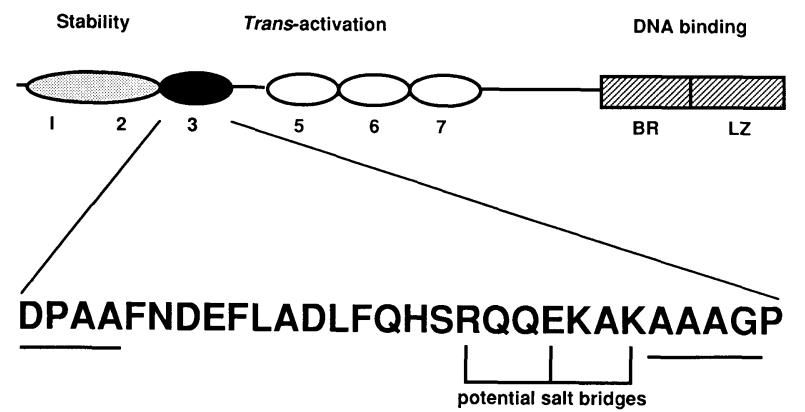

Figure 6. Schematic diagram of functional components of C/EBP. Polypeptide segments of C/EBP important for either protein stability or transcriptional activation of the albumin promoter are located at left. The DNA-binding domain, consisting of basic region and leucine zipper, is located at right. (For description of activities assigned to regions $1-2,3$, and $5-7$, see text.) The amino acid sequence of region 3 is shown at bottom. Underlined residues (DPAA and AAAG) correspond to polypeptide sequences suitable for initiating and terminating $\alpha$-helical structure. Potential salt bridges are indicated between arginine residue 86 and glutamic acid residue 89 and between glutamic acid residue 89 and lysine residue 92 .

C/EBP, region 3 conferred potent activity. The amino acid sequence of region 3 is shown in the lower part of Figure 6. At neutral $\mathrm{pH}$ this 28 -amino-acid sequence would be expected to exhibit a net charge of -1 (if unmodified). It contains a single serine residue and no threonines or tyrosines.

We offer several reasons to anticipate that region 3 might adopt an $\alpha$-helical conformation. First, it is flanked by amino acid sequences that are suitable for initiating and terminating $\alpha$-helical structure. An aspartic acid residue occurs on the immediate aminoterminal side of region 3 and is followed by a proline and two alanine residues. Such a sequence of amino acids would be suitable for initiating an $\alpha$-helix, with the aspartic acid residue forming an "N-cap" as described by Richardson and Richardson (1988). Region 3 terminates with Ala-Ala-Ala-Gly, a tetrapeptide sequence that optimally fits empirically defined criteria for termination of an $\alpha$-helix (Richardson and Richardson 1988). Finally, we note that region 3 contains oppositely charged amino acids configured in a manner suitable for the formation of salt bridges if the sequence were to adopt an $\alpha$-helical structure. Other investigators have provided evidence that activating regions occur in $\alpha$-helical (Hochschild et al. 1983) or $\alpha$-helix-permissive polypeptide segments (Giniger and Ptashne 1987; Hope et al. 1988; Yamamoto et al. 1990).

We offer a second reason to anticipate that amino-terminal regions of C/EBP will adopt ordered protein structure. Recall that deletions that removed either region 1 or region 2 failed to encode stable protein (Fig. 1B). When both of these regions were removed, stable protein was observed (Fig. 2B). These observations raise the possibility that regions 1 and 2, although not obligatorily involved in the process of trans-activation, together adopt an ordered structure. When the contiguous 1-2 region was disrupted, C/EBP appeared to be markedly destabilized. Furthermore, deletions that concomitantly lacked regions 1 and 2, such as C/EBP $\Delta 1-2$ and C/EBP $\Delta 1-3$, were expressed at levels between 5- and 10-fold greater than normal. We speculate that the folded state adopted by regions 1 and 2 may confer designed lability to C/EBP (see Fig. 6) and that truncation of either half of the region exacerbates lability. The 60 amino acids located within regions 1 and 2 contain 12 proline, 8 serine, 2 threonine, and 2 glutamic acid residues. Polypeptide regions abundant in these 4 amino acids, termed PEST sequences, have been correlated with protein lability (Rogers et al. 1986). Although we believe that the anomalous expression levels of deletion mutants in the 1-2 region are best explained by spurious effects on protein stability, we have not ruled out the possibility that these deletions affect the synthesis, processing, stability, or translation of C/EBP mRNA.

The second activating domain of C/EBP, which was located within an internal area of the protein, appeared to be composed of three redundant elements (designated regions 5-7). Removal of any one of these regions did not affect the ability of C/EBP to trans-activate the promoter of the serum albumin gene. However, when all three regions were removed, trans-activation was reduced to a level $\sim 30 \%$ that of the intact protein. Moreover, when these regions were removed from a protein that already lacked region 3, no trans-activation was observed. Previous studies of transcriptional activating domains have demonstrated functional redundancy (Hope and Struhl 1986; Gill and Ptashne 1987; Triezenberg et al. 1988). Although redundancy appears to be a common property of activating domains, we do not yet understand its significance.

Table 1 lists six parameters that help describe each of the regions that were deleted from C/EBP. These include overall length in amino acid residues, number of acidic residues, number of basic residues, number of proline residues, number of glutamine residues, and the combined number of serine and threonine residues. The four regions to which activating potential could be attributed

Table 1. Amino acid composition of segments 1-9 of C/EBP

\begin{tabular}{lcccrcc}
\hline Segment & Length & $\mathrm{D}+\mathrm{E}$ & $\mathrm{K}+\mathrm{R}$ & $\mathrm{P}$ & $\mathrm{Q}$ & $\mathrm{S}+\mathrm{T}$ \\
\hline$\Delta 1$ & 33 & 0 & 4 & 9 & 1 & 5 \\
$\Delta 2$ & 28 & 4 & 2 & 4 & 0 & 5 \\
$\Delta 3$ & 28 & 4 & 3 & 2 & 3 & 1 \\
$\Delta 4$ & 31 & 2 & 0 & 7 & 0 & 1 \\
$\Delta 5$ & 29 & 3 & 3 & 4 & 0 & 0 \\
$\Delta 6$ & 24 & 5 & 3 & 3 & 2 & 0 \\
$\Delta 7$ & 23 & 0 & 0 & 12 & 1 & 1 \\
$\Delta 8$ & 36 & 0 & 0 & 10 & 4 & 5 \\
$\Delta 9$ & 23 & 0 & 1 & 5 & 0 & 1 \\
\hline
\end{tabular}

Listed are properties of C/EBP regions designated 1-9 in Fig. 1, which include length in amino acid residues, number of acidic residues $(D+E)$, number of strongly charged basic residues $(\mathrm{K}+\mathrm{R})$, number of proline residues $(\mathrm{P})$, number of glutamine residues $(\mathrm{Q})$, and the combined number of serine and threonine residues $(\mathrm{S}+\mathrm{T})$. 
$(3,5,6$, and 7$)$ were not distinguished in charge or preponderance of any single amino acid when compared with dispensable regions $(1,2,4,8$, and 9). As such, we have been unable to assign C/EBP to any of the classes of activating domains (acidic, proline-rich, or glutaminerich/ described in earlier studies (Hope and Struhl 1986; Gill and Ptashne 1987; Ma and Ptashne 1987b; Courey and Tiian 1988; Mermod et al. 1989). Finally, we point out the possibility that C/EBP might activate gene expression indirectly. The activating domains identified in the present study may actually represent oligomerization surfaces that allow C/EBP to form higher order aggregates either with itself or with other proteins that play a more direct role in transcriptional activation. That is, we do not necessarily assume that the activating regions defined in this study are meant to interact directly with the enzymes and protein factors that mediate the process of transcription initiation. We hope that answers to this and other fundamental uncertainties concerning C/EBP will be illuminated by observations presented in this report.

\section{Materials and methods}

\section{Cell culture and transient transfection}

Monolayers of human hepatoma (HepG2) cells (Aden et al. 1979| were maintained in a $1: 1$ mixture of Dulbecco's modified Eagle medium (DMEM) and Ham's F12 medium supplemented with $10 \%$ fetal calf serum and $10 \mu \mathrm{g} / \mathrm{ml}$ insulin.

Transient transfections were carried out according to the calcium phosphate method (Graham et al. 1973). Calcium phosphate precipitates were removed $16 \mathrm{hr}$ after addition. Cells were then washed with phosphate-buffered saline (PBS) and fed with $10 \mathrm{ml}$ of culture medium. Cells were harvested $40 \mathrm{hr}$ after initial exposure to DNA. Cell lysates were prepared for either Western blot analysis or primer extension analysis. All comparisons of protein expression and trans-activation were undertaken by use of the same calcium phosphate precipitate (which was split onto two dishes) and were repeated on at least two occasions.

RNA preparation and primer extension analysis were carried out as described (Eisenberg et al. 1985). The primer used was a synthetic oligonucleotide that hybridizes between residues +56 and +80 relative to the $t k$ mRNA cap site. The major extension product is 104 nucleotides in length.

Immunofluorescence staining of C/EBP was conducted by use of cells grown on acid-etched coverslips. Cells were fixed with $1.6 \%$ paraformaldehyde in PBS for $20 \mathrm{~min}$ at room temperature. Indirect immunofluorescence was carried out as described (Friedman et al. 1988) by use of a polyclonal rabbit antiserum directed against a peptide corresponding to the carboxyterminal 15 amino acids of C/EBP (Birkenmeier et al. 1989).

Total cellular protein extracts were prepared from transfected cells for Western blot analysis, as described previously (Triezenberg et al. 1988). For electrophoretic separation of proteins, each sample was boiled for $3 \mathrm{~min}$ in sample buffer and loaded onto an $11 \%$ polyacrylamide-SDS gel (Laemmli 1970). After electrophoresis, proteins were transferred to nitrocellulose (Towbin et al. 1979) and probed by use of one of two C/EBP-specific antisera. One antiserum was directed against the carboxyterminal 15 amino acids of C/EBP and was used to detect proteins produced from all C/EBP deletion mutants (Figs. 1-3). A second antiserum was directed against a 14-amino-acid epitope located immediately amino-terminal to the C/EBP DNA- binding domain (Landschulz et al. 1988a). Antigen-antibody complexes were detected by use of ${ }^{125}$ I coupled to protein A (Amersham) and visualized by autoradiography.

\section{Recombinant plasmids}

The plasmid containing the mouse albumin promoter linked to the herpes simplex virus $t k$ gene, pAT2, has been described previously (Friedman et al. 1989). Construction of pAT2-GAL began with pAT2/ $\triangle \mathrm{DEI}$, in which the C/EBP-binding site located 100 bp upstream from the albumin mRNA cap site had been replaced by a HindIII site (Friedman et al. 1989). Two oligonucleotides were annealed to provide a 17-bp GAL4-binding site (Giniger et al. 1985) flanked by HindIII overhangs. The sequences of these oligonucleotides were 5'-AGCTCGGAAGACTCTCCTCCG-3' and 5'-AGCTCGGAGGAGAGTCTTCCG-3'. The oligonucleotides were first phosphorylated with polynucleotide kinase (New England Biolabs) and ATP. They were then annealed in $10 \mathrm{~mm}$ Tris, $1 \mathrm{~mm}$ EDTA ( $\mathrm{pH} 7.5$ ), by incubation of an equimolar mixture at $100^{\circ} \mathrm{C}$ for $3 \mathrm{~min}$, followed by successive $15-\mathrm{min}$ incubations at 68,37 , and $20^{\circ} \mathrm{C}$. The annealed, phosphorylated oligonucleotides were then ligated into the HindIII site of pAT2/DDEI (Maniatis et al. 1982).

The expression vector that encodes native C/EBP, termed pMSV-C/EBP-wt, has been described (Friedman et al. 1989). An initial set of nine deletion mutants of this plasmid were constructed by site-directed mutagenesis (Smith 1985). Oligonucleotides were designed to contain 11-15 nucleotides complementary to the two regions of the C/EBP gene, which directly flanked the segment that was to be deleted. The hexanucleotide GGTACC was included between these flanking oligonucleotides, causing each deleted segment to be replaced by a KpnI site. The end points of these nine deleted segments are provided in the legend to Figure 1. The sequences of the oligonucleotides that were used to generate the nine deletions were

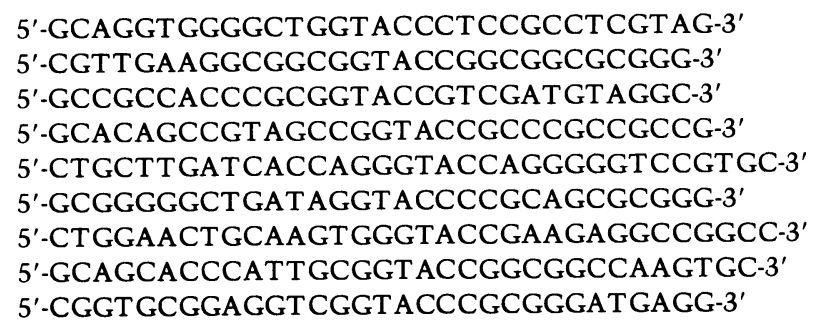

Larger deletions were prepared by recombination of the nine initial mutants, taking advantage of the fact that the introduced KpnI site of each mutant was unique to the entire variant plasmid.

Preparation of an expression vector capable of producing a hybrid protein in which the C/EBP leucine zipper was replaced with that of GCN4 made use of a bacterial expression vector termed C2G2 (Agre et al. 1989). Sequences encoding the GCN4 leucine zipper and a portion of C/EBP are contained within a MluI-HindIII restriction fragment of C2G2. Sequences encoding the analogous region of C/EBP were excised by MluI/BamHI double digestion of pMSV-C/EBP-wt/ORF, a derivative of pMSV-C/EBP-wt in which sequences encoding a portion of the 3'-untranslated region of C/EBP has been replaced with a BamHI site (S. McKnight, unpubl.). The HindIII restriction site located downstream from the protein-coding segment of C2G2 was eliminated and changed to a BamHI site by use of oligonucleotide linkers (Collaborative Research), allowing the hybrid DNA-binding domain to be appropriately positioned within C/EBP.

Preparation of expression vectors encoding hybrid proteins containing the DNA-binding domain of GAL4 and various seg- 
ments of C/EBP began with the plasmid pSG424 (Sadowski and Ptashne 1989). To construct pGAL1-2 and pGALl-3 the 240and 324-bp fragments resulting from $\mathrm{BamHI} / \mathrm{KpnI}$ digestion of $\mathrm{pC} / \mathrm{EBP} \Delta 3-9$ and $\mathrm{pC} / \mathrm{EBP} \Delta 4-9$, respectively, were ligated to the large fragment resulting from similar digestion of pSG424. To construct pGAL3, a SalI site was introduced into pC/EBP $\Delta 4$, at the amino-terminal end of region 3 , by site-directed mutagenesis. The sequence of the oligonucleotide used to introduce this restriction site was 5'-GAAGGCGGCCGGTCGACTGTAGGCGCTGATG-3'. The resulting plasmid was doubly digested with SalI and KpnI, releasing an 86-bp fragment, which was ligated to pSG424 after digestion with the same two restriction enzymes, yielding pGAL3. Preparation of pGAL1-9, pGAL3-9, and pGAL4-9 began with a derivative of pSG424, pGALM, containing a modified polylinker, which contains an NcoI site (G. Kato and C. Dang, unpubl.). Preparation of these three plasmids also made use of pMSV-C/EBP-B9C, a derivative of pMSV-C/EBP-wt in which a BamHI site replaces the nucleotides that encode amino acids 270 and 271 (S. McKnight, unpubl.). This BamHI site was first changed to an $\mathrm{XbaI}$ site by use of oligonucleotide linkers (Collaborative Research). Digestion of this plasmid with $\mathrm{NcoI}$ and $\mathrm{XbaI}$ released an 810-bp fragment. This fragment was ligated to the large fragment resulting from similar digestion of pGALM, yielding pGAL1-9. Finally, to create pGAL3-9, and pGAL4-9, the 390- and 310-bp fragments released by double digestion of C/EBP $\Delta 1-2$ and C/EBP $\Delta 1-3$ with $N c o I$ and MluI were individually ligated to the large fragment released by double digestion of pGAL1-9 with the same enzymes.

\section{Acknowledgments}

We thank Eve Walton for assistance in constructing the initial set of C/EBP deletion mutants, Mark Ptashne for pSG424, Chi Dang for pGALM, Charles Vinson for pointing out the potential helical structure of region 3, Bob Kingsbury for technical assistance, Christine Norman for clerical assistance, and our many colleagues at the Carnegie Embryology Department for advice and encouragement. ADF was supported by a Physician Scientist Award from the National Institutes of Health (NIH). This work was otherwise funded by the Howard Hughes Medical Research Institute and the Carnegie Institution of Washington.

The publication costs of this article were defrayed in part by payment of page charges. This article must therefore be hereby marked "advertisement" in accordance with 18 USC section 1734 solely to indicate this fact.

\section{References}

Aden, D.P., A. Fogel, S. Plotkin, I. Damjanov, and B.B. Knowles. 1979. Controlled synthesis of $\mathrm{HBsAg}$ in a differentiated human liver carcinoma-derived cell line. Nature 282: 615618.

Agre, P., P.F. Johnson, and S.L. McKnight. 1989. Cognate DNA binding specificity retained after leucine zipper exchange between GCN4 and C/EBP. Science 246: 922-926.

Birkenmeier, E.H., B. Gwynn, S. Howard, J. Jerry, J.I. Gordon, W.H. Landschulz, and S.L. McKnight. 1989. Tissue-specific expression, developmental regulation and genetic mapping of the gene encoding C/EBP. Genes Dev. 3: 1146-1156.

Bohmann, D. and R. Tjian. 1989. Biochemical analysis of transcriptional activation by Jun: Differential activity of c- and v-Jun. Cell 59: 709-717.

Cereghini, S., M. Raymondjean, A.G. Carranca, P. Herbomel, and M. Yaniv. 1987. Factors involved in control of tissuespecific expression of albumin gene. Cell 50: 627-638.

Costa, R.H., D.R. Grayson, K.G. Xanthopoulos, and J.E. Dar- nell, Jr. 1988. A liver-specific DNA binding protein recognizes multiple nucleotide sites in regulatory regions of transthyretin, alpha 1-antitrypsin, albumin, and SV40 genes. Proc. Natl. Acad. Sci. 85: 3840-3844.

Courey, A.J. and R. Tjian. 1988. Analysis of Spl in vivo reveals multiple transcriptional domains, including a novel glutamine-rich activation motif. Cell 55: 887-898.

Eisenberg, S.P., D.M. Coen, and S.L. McKnight. 1985. Promoter domains required for expression of plasmid-borne copies of the herpes simplex virus thymidine kinase gene in virus-infected mouse fibroblasts and microinjected frog oocytes. Mol. Cell. Biol. 5: 1940-1947.

Friedman, A.D., S.J. Triezenberg, and S.L. McKnight. 1988. Expression of a truncated viral trans-activator selectively impedes lytic infection by its cognate virus. Nature 335: 452454.

Friedman, A.D., W.H. Landschulz, and S.L. McKnight. 1989. CCAAT/enhancer binding protein activates the promoter of the serum albumin gene in cultured hepatoma cells. Genes Dev. 3: 1314-1322.

Gill, G. and M. Ptashne. 1987. Mutants of GAL4 protein altered in an activation function. Cell 51: 121-126.

Giniger, E. and M. Ptashne. 1987. Transcription in yeast activated by a putative amphipathic $\alpha$-helix linked to a DNA binding unit. Nature 330: 670-672.

Giniger, E., S.M. Varnum, and M. Ptashne. 1985. Specific DNA binding of GAL4, a positive regulatory protein of yeast. Cell 40: 767-774.

Graham, F.L. and A.J. Van der Eb. 1973. A new technique for the assay of infectivity of human adenovirus 5 DNA. Virology 52: 456-457.

Graves, B., P.F. Johnson, and S.L. McKnight. 1986. Homologous recognition of a promoter domain common to the MSV LTR and the HSV tk gene. Cell 44: 565-576.

Hochschild, A., N. Irwin, and M. Ptashne. 1983. Repressor structure and the mechanism of positive control. Cell 32: 319-325.

Hope, I.A. and K. Struhl. 1986. Functional dissection of a eukaryotic transcriptional activator protein, GCN4 of yeast. Cell 46: 885-894.

Hope, I.A., S. Mahadevan, and K. Struhl. 1988. Structural and functional characterization of the short acidic transcriptional activation region of yeast GCN4 protein. Nature 333: $635-640$.

Johnson, P.F., W.H. Landschulz, B.J. Graves, and S.L. McKnight. 1987. Identification of a rat liver nuclear protein that binds to the enhancer core element of three animal viruses. Genes Dev. 1: 133-146.

Kaestner, K.H., R.J. Christy, and M.D. Lane. 1990. Mouse insulin-responsive glucose transporter gene: Characterization of the gene and trans-activation by the CCAAT/enhancer binding protein. Proc. Natl. Acad. Sci. 87: 251-255.

Keegan, L., G. Gill, and M. Ptashne. 1986. Separation of DNA binding from the transcription-activating function of a eukaryotic regulatory protein. Science 231: 699-704.

Laemmli, U.D. 1970. Cleavage of structural proteins during the assembly of the head of bacteriophage T4. Nature 227: 680685.

Landschulz, W.H., P.F. Johnson, E.Y. Adashi, B.J. Graves, and S.L. McKnight. 1988a. Isolation of a recombinant copy of the gene encoding C/EBP. Genes Dev. 2: 786-800.

Landschulz, W.H., P.F. Johnson, and S.L. McKnight. 1988b. The leucine zipper: A hypothetical structure common to a new class of DNA binding proteins. Science 240: 1759-1764.

-1989. The DNA binding domain of the rat liver nuclear protein C/EBP is bipartite. Science 243: $1681-1688$. 
Laughon, A. and R.G. Gesteland. 1984. Primary structure of the Saccharomyces cerevisiae GAL4 gene. Mol. Cell. Biol. 4: 260-267.

Ma, J. and M. Ptashne. 1987a. Deletion analysis of GAL4 defines two transcriptional activating segments. Cell 48: 847853.

- 1987b. A new class of yeast transcriptional activators. Cell 51: 113-119.

Maire, P., J. Wuarin, and U. Schibler. 1989. The role of cisacting promoter elements in tissue specific albumin gene expression. Science 244: 343-346.

Maniatis, T., E.F. Fritsch, and J. Sambrook. 1982. Molecular cloning: A laboratory manual. Cold Spring Harbor Laboratory Press, Cold Spring Harbor, New York.

McKnight, S.L., M.D. Lane, and S. Gluecksohn-Waelsch. 1989. Is CCAAT/enhancer-binding protein a central regulator of energy metabolism? Genes Dev. 3: 2021-2024.

Mermod, N., E.A. O'Neill, T.J. Kelly, and R. Tjian. 1989. The proline-rich transcriptional activator of CTF/NF-1 is distinct from the replication and DNA binding domain. 1989. Cell 58: 741-753.

Park, E.A., W.J. Roesler, J. Liu, D.J. Klemm, A.L. Gurney, J.D. Thatcher, J. Shuman, A. Friedman, and R.W. Hanson. 1990. The role of the CCAAT/enhancer binding protein in the transcriptional regulation of the gene for phosphoenolpyruvate carboxykinase (GTP). Mol. Cell. Biol. (in press).

Richardson, J.S. and D.C. Richardson. 1988. Amino acid preferences for specific locations at the ends of $\alpha$ helices. Science 240: $1648-1652$.

Rogers, S., R. Wells, and M. Rechsteiner. 1986. Amino acid sequences common to rapidly degraded protein: The PEST hypothesis. Science 234: 364-368.

Sadowski, I. and M. Ptashne. 1989. A vector for expressing GAL4(1-147) fusions in mammalian cells. Nucleic Acids Res. 17: 7539 .

Smith, M. 1985. In vitro mutagenesis. Annu. Rev. Genet. 19: 423-463.

Tanaka, M. and W. Herr. 1990. Differential transcriptional activation by Oct-1 and Oct-2: Interdependent activation domains induce Oct-2 phosphorylation. Cell 60: 375-386.

Towbin, H., T. Staehelin, and J. Gerdon. 1979. Electrophoretic transfer of proteins from polyacrylamide gels to nitrocellulose sheets: procedure and some applications. Proc. Natl. Acad. Sci. 76: 4350-4354.

Triezenberg, S.J., R.C. Kingsbury, and S.L. McKnight. 1988. Functional dissection of VP16, the trans-activator of herpes simplex virus immediate early gene expression. Genes Dev. 2: 718-729.

Trus, M., N. Benvenisty, H. Cohen, and L. Reshef. 1990. Developmentally regulated interactions of over nuclear factors with the rat phosphoenolpyruvate carboxykinase promoter. Mol. Cell. Biol. 10: 2418-2422.

Vinson, C.R., P.B. Sigler, and S.L. McKnight. 1989. Scissors-grip model for DNA recognition by a family of leucine zipper proteins. Science 246: 911-916.

Yamamoto, K.K., G.A. Gonzalez, P. Menzel, J. Rivier, M.R. Montminy. 1990. Characterization of a bipartite activator domain in transcription factor CREB. Cell 60: 611-617. 


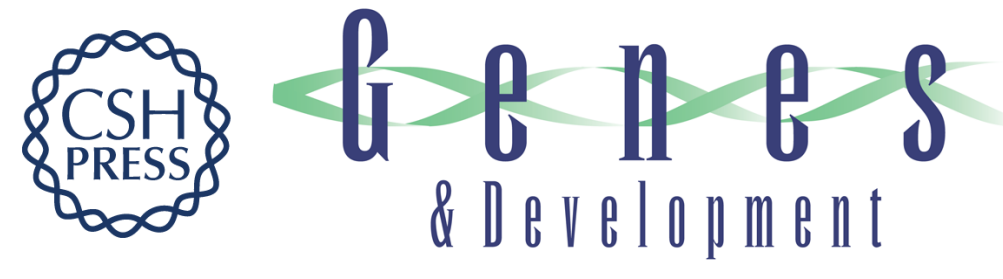

\section{Identification of two polypeptide segments of CCAAT/enhancer-binding protein required for transcriptional activation of the serum albumin gene.}

A D Friedman and S L McKnight

Genes Dev. 1990, 4:

Access the most recent version at doi:10.1101/gad.4.8.1416

References This article cites 41 articles, 20 of which can be accessed free at: http://genesdev.cshlp.org/content/4/8/1416.full.html\#ref-list-1

License

Email Alerting Receive free email alerts when new articles cite this article - sign up in the box at the top Service right corner of the article or click here.

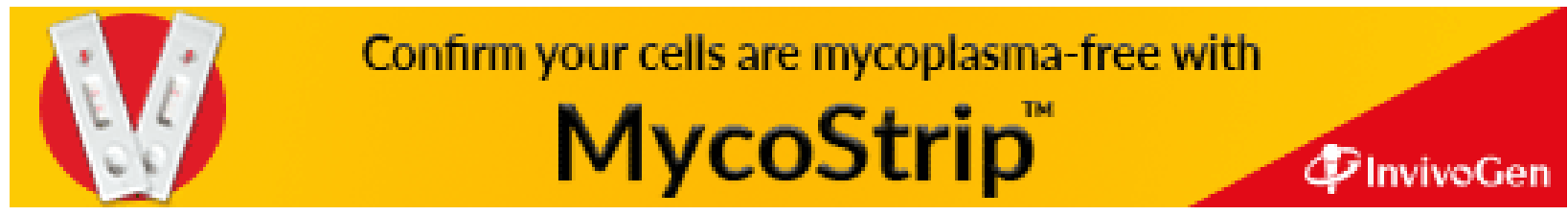

